\title{
Correction to: Advanced Practice Nursing in Africa
}

\author{
Nelouise Geyer and Christmal Dela Christmals
}

\section{Correction to: S. B. Hassmiller, J. Pulcini (eds.), Advanced Practice Nursing Leadership: A Global Perspective, Advanced Practice in Nursing, https://doi.org/10.1007/978-3-030-20550-8_6}

An incorrect data and information have been used in Chapter 6 on page 67. A correct data has been updated as below:

An earlier one-year certificate educational program introduced the family practice role in Swaziland between 1979-1995. This program was presented through a partnership between University of Swaziland and Denmark. Various barriers to this programme contributed to the decision to develop the family practice role at a master's level as an advanced practice nurse practitioner role. The PEPPA framework (Participatory, Evidence-based, Patient focused Process for Advanced practice nursing) (Bryant-Lukosius and DiCenso 2004) provided the context for the development and implementation of the nurse practitioner role in Eswatini starting with stakeholder consultations in 2004-2007. This was followed by role definition, educational preparation and policy/regulatory requirements including the development of a scope of practice. The curriculum was approved by the university in 2016 and the first students were enrolled in 2017. The Global Health Service Partnership (GHSP: a partnership between US Peace Corps, PEPFAR, and Seed Global Health) provided experienced nurse practitioner faculty for a year to support the family practice nursing program until a core family practice nurse faculty could be developed. One GSHP faculty stayed on as program coordinator to provide continuity and expertise to fully develop the program (Dalmini et al. 2020).

The updated online version of this chapter can be found at https://doi.org/10.1007/ 978-3-030-20550-8_6 